\title{
Force measurements in stiff, 3D, opaque granular materials
}

\author{
Ryan C. Hurley ${ }^{1, \star}$, Stephen A. Hall ${ }^{2}$, José E. Andrade ${ }^{3}$, and Jonathan Wright ${ }^{4}$ \\ ${ }^{1}$ Lawrence Livermore National Laboratory, Livermore, CA 94550, USA \\ ${ }^{2}$ Division of Solid Mechanics, Lund University, Lund 22100, Sweden \\ ${ }^{3}$ California Institute of Technology, Pasadena, CA 91125, USA \\ ${ }^{4}$ European Synchrotron Radiation Facility, Grenoble 38000, France
}

\begin{abstract}
We present results from two experiments that provide the first quantification of inter-particle force networks in stiff, 3D, opaque granular materials. Force vectors between all grains were determined using a mathematical optimization technique that seeks to satisfy grain equilibrium and strain measurements. Quantities needed in the optimization - the spatial location of the inter-particle contact network and tensor grain strains were found using 3D X-ray diffraction and X-ray computed tomography. The statistics of the force networks are consistent with those found in past simulations and 2D experiments. In particular, we observe an exponential decay of normal forces above the mean and a partition of forces into strong and weak networks. In the first experiment, involving 77 single-crystal quartz grains, we also report on the temporal correlation of the force network across two sequential load cycles. In the second experiment, involving 1099 single-crystal ruby grains, we characterize force network statistics at low levels of compression.
\end{abstract}

\section{Introduction}

The stress, stability, and transport properties of granular materials are controlled by the network of contact forces between grains (e.g., [1, 2]). Researchers have studied these networks for decades in an effort to build multiscale models for granular media. However, experimental capabilities for measuring forces in stiff, 3D, frictional materials remain limited. Such data are required to validate models and provide access to broader ranges of materials and environments than are currently available in computations.

Photoelastic measurements on 2D disk assemblies provided the earliest visualizations of force networks in granular solids and continue to furnish new insight into the relationship between these networks and mechanical properties [3]. Measurements in 3D systems include those using microscopy with emulsion droplets [4], X-ray computed tomography (XRCT) with rubber spheres [5], and laser scanning microtomography with hydrogels [6]. To move beyond the spherical, frictionless, soft 3D grains explored in past experiments, XRCT and 3D X-ray diffraction (3DXRD) have recently been combined to explore the contact network and grain strains in stiff, 3D, frictional grains $[7,8]$. Recent work has also enabled the calculation of the full force network in these materials from the XRCT and 3DXRD data [9].

In this paper, we discuss the force measurement capabilities originally described in [9] in the context of two experiments. Both experiments used XRCT and 3DXRD to furnish the contact network and tensor grain strains needed

\footnotetext{
^e-mail: hurley10@11nl.gov
}

to infer inter-particle forces in a compressed granular material. In the first experiment, 77 single-crystal quartz grains were subjected to two compression cycles. We discuss force network statistics and correlation across load cycles. In the second experiment, 1099 ruby grains were subjected to three compression cycles. We discuss preliminary force network inference for this experiment.

\section{Experiments}

Both experiments described in this paper were performed at beam line ID11 of the European Synchrotron Radiation Facility (ESRF). The experiments used a custom-built loading device [9] to compress granular samples in the beam line, as shown in Fig. 1a. In the first experiment, described in more detailed in [9], 77 nearly spherical singlecrystal quartz grains with diameters ranging from 220 to $310 \mu \mathrm{m}$ were compressed through two load cycles. In the second experiment, 1099 nearly spherical single-crystal ruby grains with diameters ranging from 100 to $120 \mu \mathrm{m}$ were compressed through four load cycles. In each experiment, at load increments of 5 to $30 \mathrm{~N}$, strain was held constant while the granular sample was fully illuminated by a $1.5 \mathrm{~mm}$ wide by $1.5 \mathrm{~mm}$ tall incident X-ray beam and rotated, in small increments, over $180^{\circ}$ for 3DXRD and transmission radiography scans. 3DXRD data was analyzed using techniques from [10] to determine average tensor grain strains, $\overline{\boldsymbol{\epsilon}}_{\alpha}$, for each grain, $\alpha$, at each load increment. Average grain stresses, $\overline{\boldsymbol{\sigma}}_{\alpha}$, were computed using the elastic stiffness constants, $C_{i j}$, of singlecrystal quartz from [11] and single-crystal ruby from [12]. 

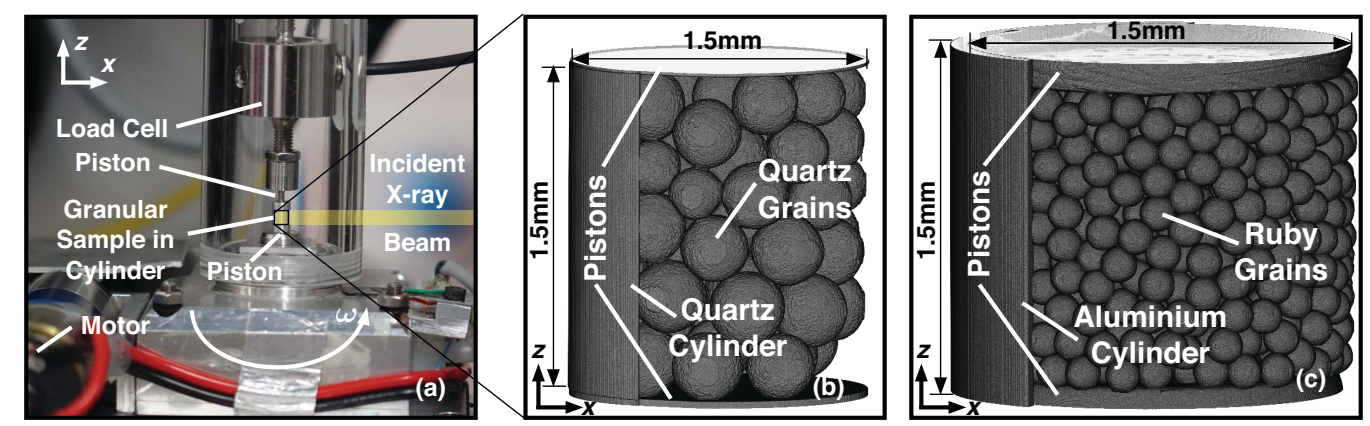

Figure 1. (a) Custom-built loading device positioned in beam line ID11 at ESRF, showing orientation of incident X-ray beam, rotation direction, and laboratory coordinate frame. (b) XRCT image of 77 single-crystal quartz grains in an amorphous quartz cylinder (also reported in [9]). (c) XRCT image of 1099 single-crystal ruby grains in an aluminium cylinder.

Transmission radiography images were used to construct 3D XRCT images of the first and second experiments using the ASTRA [13] and PyHST2 [14] toolboxes, respectively, with a resolution of approximately $1.5 \mu \mathrm{m}$. These images were processed using algorithms such as binarization and topological watershed in Matlab to obtain Figs. $1 \mathrm{~b}$ and $1 \mathrm{c}$, showing segmented grains. From these images, grain volumes and centroids were determined. Contact locations were determined by first finding all pixels whose 26-pixel neighborhood contained a pixel belonging to distinct grains (or a grain and a boundary), before thresholding to retain only pixel clusters with volume greater than $0.003 \%$ of the smallest grain in the assembly. Results were not sensitive to this threshold as long as it was small.

\section{Force inference}

Force vectors were inferred at every inter-particle contact point, at each load step, in each experiment using the measurements from 3DXRD and XRCT as inputs into the multi-objective optimization procedure from [9]. This optimization procedure seeks to satisfy grain equilibrium and stress measurements by solving

$$
\boldsymbol{f}=\underset{\quad}{\arg \min \left(\left|\boldsymbol{K}_{s} \boldsymbol{f}-\boldsymbol{b}_{\boldsymbol{s}}\right|_{2}+\lambda\left|\boldsymbol{K}_{e} \boldsymbol{f}\right|_{2}\right)}
$$

where $\boldsymbol{f}$ contains all force vectors, $\boldsymbol{K}_{s}$ and $\boldsymbol{K}_{e}$ contain contact point locations, $\mu$ is the friction coefficient $(0.7$ for quartz [15], 0.1 for ruby-ruby and 0.4 for ruby-aluminum [12]), $\boldsymbol{b}_{s}$ contains volume-averaged grain stress tensor components multiplied by grain volumes, $\boldsymbol{f}_{n}$ contains all normal force magnitudes, $\boldsymbol{f}_{t}$ contains all shear force magnitudes, $\lambda$ is a tradeoff parameter, and $|\cdot|$ represents the 2-norm. The first 2-norm contains all equations relating volume-averaged stress to boundary forces on each grain, or $V_{\alpha} \bar{\sigma}_{i j}^{(\alpha)}=\sum_{\beta=1}^{N_{c}^{\alpha}} x_{i}^{(\beta)} \otimes f_{j}^{(\beta)}$, where $N_{c}^{\alpha}$ is the number of contacts on grain $\alpha$. The second 2-norm contains equations of linear and angular force balance for each grain. Forces that minimize these terms are those that most closely satisfy balance of forces while also producing grain stresses that most closely agree with 3DXRD measurements. The constraint requires all inter-particle forces to be positive and obey a Coulomb friction constraint. Solutions to Eq. (1) are unique (determinate) when $3 N_{c}^{T} \leq 12 N_{p}^{T}$, where $N_{c}^{T}$ is the total number of contacts and $N_{p}^{T}$ is the total number of grains in the sample. This condition is fulfilled for both samples examined here and will generally be fulfilled for systems with a coordination number close to 8 if the number of grain-grain contacts significantly exceeds the number of grain-boundary contacts. Full solution details are given in [9].

We note that the high moduli of the materials studied $\left(E_{33} \approx 103 \mathrm{GPa}\right.$ for quartz; $E_{33} \approx 495 \mathrm{GPa}$ for ruby) limit relative grain deformation to less than 5 pixels for quartz and less than 1 pixel for ruby at maximum sample compression and thus prohibit the use of local deformation as a means for inferring accurate normal contact forces from a known contact law.

\section{Experiment 1: 77 quartz grains}

The load cell data recorded during the first experiment are shown in Fig. 2a and illustrates that the sample of 77 quartz grains was subjected to two compression cycles. Figure $2 \mathrm{~b}$ shows the evolution of volume-averaged grain stress for the sample, $\bar{\sigma}$, obtained by computing $\overline{\boldsymbol{\sigma}}=\frac{1}{V} \sum_{\alpha=1}^{77} \overline{\boldsymbol{\sigma}}_{\alpha} V_{\alpha}$, where $V_{\alpha}$ is the volume of grain $\alpha$ and $V$ is the total volume (including pore space) of the granular sample between loading pistons. We observe a consistent trend between $\bar{\sigma}$ components and the load cell data. We also observe that $\bar{\sigma}_{z z}$ is greater than both $\bar{\sigma}_{x x}$ and $\bar{\sigma}_{y y}$ throughout most of the experiment; the lateral pressure coefficients, $k_{0}^{(1)}=\bar{\sigma}_{z z} / \bar{\sigma}_{x x}$ and $k_{0}^{(2)}=\bar{\sigma}_{z z} / \bar{\sigma}_{y y}$, are less than unity. However, $k^{(1)}>1$ and $k^{(2)}>1$ when the sample is fully unloaded, implying that a residual stress is presented in the system, a feature sometimes found in overconsolidated soils.

Results from force inference, using Eq. (1), for load steps 15 and 24 are illustrated in Fig. 3a and 3b, in which force chain structures can clearly be observed. Figure $2 \mathrm{c}$ shows the sum of vertical forces on the bottom, top and side boundaries. The sums exhibit the same trend as the load cell data in Fig. 2a, but are lower in magnitude due to friction between the load piston and quartz cylinder [9]. Figure $2 \mathrm{~d}$ illustrates the mean, maximum, and maximum 


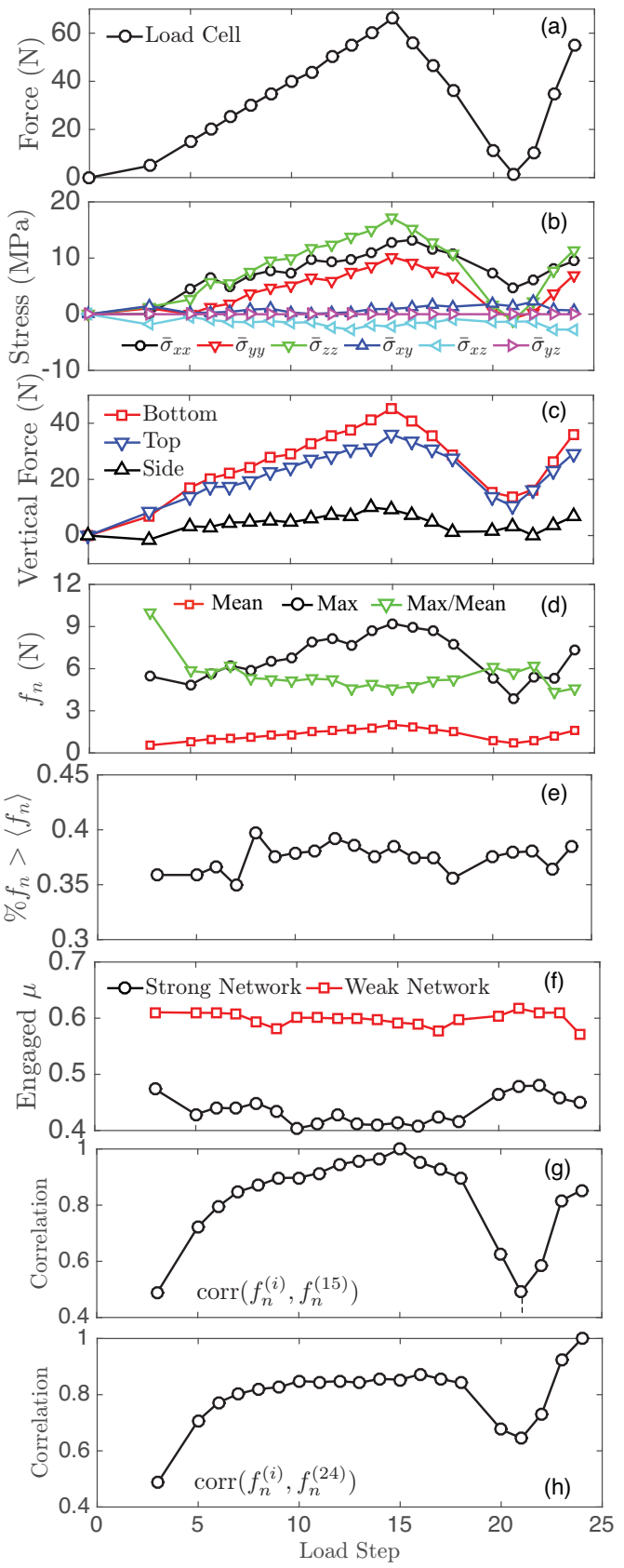

Figure 2. Results of first experiment. (a) Load cell data. (b) Volume-averaged stress evolution. (c) Sum of inferred vertical forces on bottom, top and side boundaries. (d) Statistics of inferred normal forces. (e) Percentage of normal forces above the mean. (f) Engaged friction in strong and weak force networks. (g) Correlation of entire force network with force network at load step 15. (h) Same as (g) but for load step 24.

divided by mean normal force magnitude, $f_{n}$, in the entire force network. The mean and maximum values of $f_{n}$ exhibit the same trend as the applied load while the maximum divided by mean exhibits the inverse trend. This observation suggests that the largest values of $f_{n}$ in the system are closer to the mean at high loads, implying a more homogeneous response. This finding was corroborated by a decreasing value (with increasing load) of the force distribution exponent and the Gini coefficient in [9].

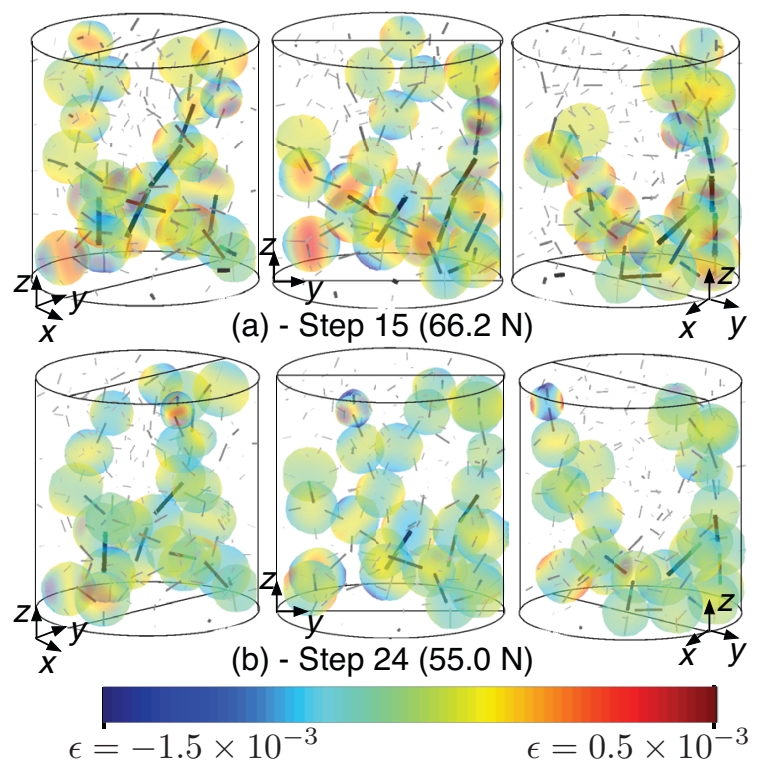

Figure 3. (a) Three views of the force network at load step 15. All forces are plotted as lines but only grains containing a normal force satisfying $f_{n} \geq 2\left\langle f_{n}\right\rangle$ are shown for clarity. Grains are $30 \%$ opaque and plotted with strain ellipsoids $[8,9]$. (b) Same as (a) but for load step 24.

Figure 2e illustrates that the force network can be partitioned at each load step into a strong network $\left.\left(f_{n}\right\rangle\left\langle f_{n}\right\rangle\right)$ containing about $38 \%$ of contacts and a weak network $\left(f_{n} \leq\left\langle f_{n}\right\rangle\right)$ containing about $62 \%$ of contacts, which is quantitatively consistent with simulations [1]. Fig. 2f illustrates that the weak network features a higher value of engaged friction, $\left\langle f_{t} /\left(\mu f_{n}\right)\right\rangle$, than the strong network, also consistent with simulations [16] and suggesting that the weak network dissipates more energy in the system.

To explore force network reversibility and the influence of structural packing features on forces, we compute the correlation coefficient of forces across load steps by

$$
\operatorname{corr}\left(\boldsymbol{f}_{n}^{(i)}, \boldsymbol{f}_{n}^{(j)}\right)=\frac{\sum_{k=1}^{N_{c}}\left(\boldsymbol{f}_{k}^{(i)}-\left\langle\boldsymbol{f}^{(i)}\right\rangle\right)\left(\boldsymbol{f}_{k}^{(j)}-\left\langle\boldsymbol{f}^{(j)}\right\rangle\right)}{\sum_{k=1}^{N_{c}}\left(\boldsymbol{f}_{k}^{(i)}-\left\langle\boldsymbol{f}^{(i)}\right\rangle\right)^{2}\left(\boldsymbol{f}_{k}^{(j)}-\left\langle\boldsymbol{f}^{(j)}\right\rangle\right)^{2}}
$$

where $\boldsymbol{f}^{(i)}$ is a an ordered vector containing all values of $f_{n}$ for step $i, N_{c}$ is the number of contacts in all load steps, and forces are 0 if their corresponding contact does not exist in a given step. We observe in Fig. $2 \mathrm{~g}$ that the force network at peak load, step 15 , is highly correlated (corr $>0.75$ ) to previous steps after step 5 . This suggests that only minor relative changes between forces occur as the applied load increases from $1 / 3$ of its peak to its peak. Furthermore, we observe in Fig. $2 \mathrm{~h}$ that the force networks in steps near the first peak load (steps 7 through 18) are highly correlated with the network at the second peak load (step 24 ), indicating a significant amount of reversibility in the force network across load cycles. We suspect that these trends occur because the force network is determined by the structural features of the packing, which change minimally throughout the experiment in this confined sample. 


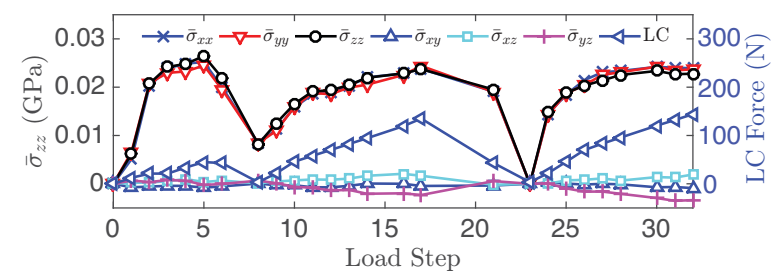

Figure 4. Load cell (LC) and grain stress data from second experiment. Steps with missing data points

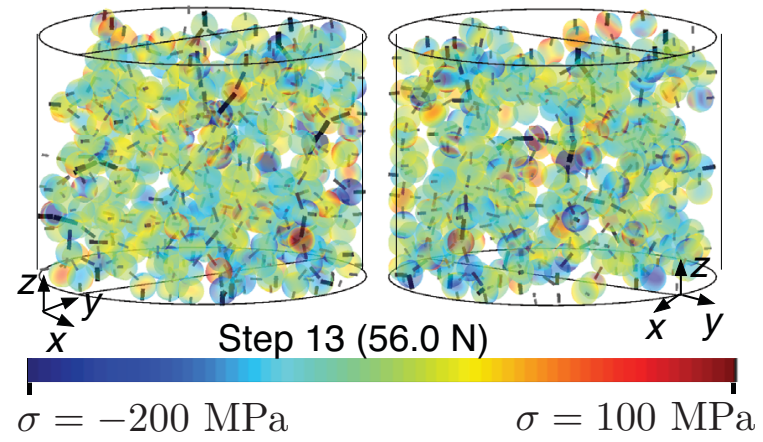

Figure 5. Two views of the force network at load step 13. Only forces for which $f_{n} \geq 2\left\langle f_{n}\right\rangle$ and corresponding grains are shown for clarity. Grains are $30 \%$ opaque and plotted with stress ellipsoids $[8,9]$.

\section{Experiment 2: 1099 ruby grains}

The load cell and grain stress data from the second experiment are shown in Fig. 4 and illustrate that the sample of 1099 ruby grains was subjected to three compression cycles. During the final compression cycle, the grains were compacted until widespread fracture occurred. We observe that the stress $\bar{\sigma}_{z z}$ along the compression axis falls below $\bar{\sigma}_{x x}$ and $\bar{\sigma}_{y y}$ after the first load cycle. This behavior was also seen during unload in the first experiment and suggests a residual lateral stress is presented in the system.

Results from force inference using Eq. (1) for load step 13 is illustrated in Fig. 5. Force chain structures are visually observed in these figures but we leave their full quantification for future publication. Here, we report that the normal forces very closely exhibit an exponential decay above the mean and partition into strong and weak networks, with the strong network $\left.\left(f_{n}\right\rangle\left\langle f_{n}\right\rangle\right)$ containing approximately $42 \%$ of all contact points.

\section{Discussion and conclusions}

We have presented results from two experiments that provide the first measurements of inter-particle force networks in stiff, 3D, frictional and opaque granular materials. The results demonstrate the potential for the approach to validate multiscale models for granular media and explore a broader range of granular material responses (e.g., fracture and communition) than is currently available computationally. Ongoing analysis of data generated from larger assemblies will shed light on local stability and rearrangements around grain fractures or strain localizations.

\section{Acknowledgements}

The authors acknowledge the ESRF for synchrotron beam time for proposals ma1913 and ma2665. R.C.H. and J.E.A acknowledge U.S. AFOSR Grant No. FA9550-12-1-0091 and U.S. DTRA Grant No. HDTRA1-12-1-0041. S.A.H. acknowledges a Marie Curie FP7 integration grant within the 7th EU Framework Programme. R.C.H. acknowledges support from Lawrence Livermore National Laboratory's Lab (LLNL) Directed Research and Development program under grant 17-LW-009. Part of this work was performed under the auspices of the U.S. Department of Energy by LLNL under Award No. DE-AC52-07NA27344.

\section{References}

[1] F. Radjai, D.E. Wolf, M. Jean, J.J. Moreau, Physical review letters 80, 61 (1998)

[2] B. Gilles, C. Coste, Physical review letters 90, 174302 (2003)

[3] T.S. Majmudar, R.P. Behringer, Nature 435, 1079 (2005)

[4] J. Brujić, S.F. Edwards, I. Hopkinson, H.A. Makse, Physica A: Statistical Mechanics and its Applications 327, 201 (2003)

[5] M. Saadatfar, A.P. Sheppard, T.J. Senden, A.J. Kabla, Journal of the Mechanics and Physics of Solids 60, 55 (2012)

[6] N. Brodu, J.A. Dijksman, R.P. Behringer, Nature communications 6 (2015)

[7] K. Alshibli, M.B. Cil, P. Kenesei, U. Lienert, Granular matter 15, 517 (2013)

[8] S. Hall, J. Wright, Géotechnique Letters 5, 236 (2015)

[9] R. Hurley, S. Hall, J. Andrade, J. Wright, Physical Review Letters 117, 098005 (2016)

[10] J. Oddershede, S. Schmidt, H.F. Poulsen, H.O. Sørensen, J. Wright, W. Reimers, Journal of Applied Crystallography 43, 539 (2010)

[11] P. Heyliger, H. Ledbetter, S. Kim, The Journal of the Acoustical Society of America 114, 644 (2003)

[12] E.R. Dobrovinskaya, L.A. Lytvynov, V. Pishchik, Sapphire: material, manufacturing, applications (Springer Science \& Business Media, 2009)

[13] W.J. Palenstijn, K.J. Batenburg, J. Sijbers, The ASTRA tomography toolbox, in 13th International Conference on Computational and Mathematical Methods in Science and Engineering, CMMSE (2013), Vol. 2013

[14] A. Mirone, E. Brun, E. Gouillart, P. Tafforeau, J. Kieffer, Nuclear Instruments and Methods in Physics Research Section B: Beam Interactions with Materials and Atoms 324, 41 (2014)

[15] J. Byerlee, Pure and applied geophysics 116, 615 (1978)

[16] N. Guo, J. Zhao, Computers and Geotechnics 47, 1 (2013) 